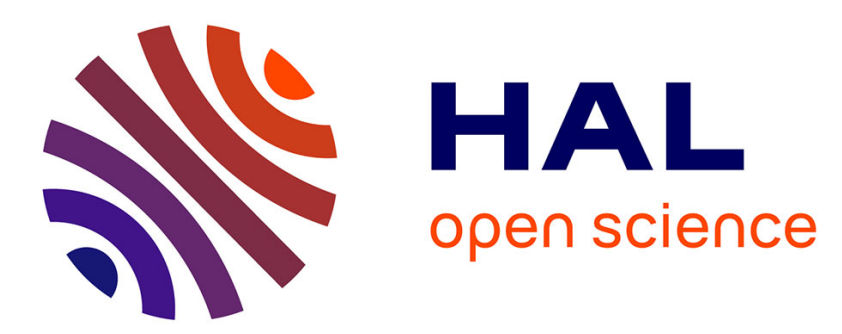

\title{
Finite-SNR Diversity-Multiplexing Tradeoff for Spatially Correlated Rayleigh MIMO Channels
}

Ammar El Falou, Charlotte Langlais, Walaa Hamouda, Charbel Abdel Nour, Catherine Douillard

\section{- To cite this version:}

Ammar El Falou, Charlotte Langlais, Walaa Hamouda, Charbel Abdel Nour, Catherine Douillard. Finite-SNR Diversity-Multiplexing Tradeoff for Spatially Correlated Rayleigh MIMO Channels. ICC 2014 : IEEE International Conference on Communications, Jun 2014, Sidney, Australia. pp.5604-5609, 10.1109/ICC.2014.6884214 . hal-01015125

\section{HAL Id: hal-01015125 \\ https://hal.science/hal-01015125}

Submitted on 17 Feb 2020

HAL is a multi-disciplinary open access archive for the deposit and dissemination of scientific research documents, whether they are published or not. The documents may come from teaching and research institutions in France or abroad, or from public or private research centers.
L'archive ouverte pluridisciplinaire HAL, est destinée au dépôt et à la diffusion de documents scientifiques de niveau recherche, publiés ou non, émanant des établissements d'enseignement et de recherche français ou étrangers, des laboratoires publics ou privés. 


\title{
Finite-SNR Diversity-Multiplexing Tradeoff for Spatially Correlated Rayleigh MIMO Channels
}

\author{
Ammar El Falou ${ }^{\dagger}$, Charlotte Langlais ${ }^{\dagger}$, Walaa Hamouda ${ }^{\ddagger}$, Charbel Abdel Nour ${ }^{\dagger}$ and Catherine Douillard ${ }^{\dagger}$ \\ †Institut Mines-Telecom; Telecom Bretagne; UMR CNRS 3192 Lab-STICC \\ Electronics Department, Technopôle Brest Iroise CS 83818, 29238 Brest Cedex 3 \\ $\ddagger$ Concordia University, Department of Electrical and Computer Engineering \\ Montreal, Quebec, H3G 1M8, Canada \\ Email: \{ammar.elfalou, charlotte.langlais, charbel.abdelnour, catherine.douillard\}@telecom-bretagne.eu, \\ hamouda@ece.concordia.ca
}

\begin{abstract}
In this paper, we derive the exact expression of the finite-signal to noise ratio (SNR) diversity-multiplexing tradeoff (DMT) for Rayleigh fading multiple-input multiple-output (MIMO) channels with dual correlated antennas at the transmitter $\left(2 \times N_{r}\right)$ and/or at the receiver $\left(N_{t} \times 2\right)$. We first derive the exact outage probability versus SNR. While finite-SNR DMT and outage probabilities are usually only estimated, we show that the numerical results of our derived outage probability and finiteSNR DMT are identical to those obtained using Monte Carlo simulations. Furthermore, it is shown that achievable diversity gains at realistic SNRs are significantly lower than asymptotic values and that the DMT degrades as the spatial correlation increases. Space-time codes (STCs) for MIMO systems are conventionally designed to achieve the asymptotic DMT frontier. This finite-SNR DMT could provide a new insight to design STCs for practical MIMO systems optimized at realistic SNRs and propagation environments.
\end{abstract}

\section{INTRODUCTION}

Most of the modern wireless communication systems as WiMAX, WiFi, LTE and 4G have adopted the use of multiple antennas at the transmitter and the receiver, called multipleinput multiple-output (MIMO). Indeed, MIMO coding potential benefits are data rate increase and better reliability. Spatial multiplexing techniques [1] have been incorporated for data rate increase whereas space-time codes (STCs) [2] have been designed to improve the channel reliability through spatial diversity. Multiplexing and diversity gains can be simultaneously delivered with a fundamental tradeoff between them [3]. The diversity-multiplexing tradeoff (DMT) defines the optimal tradeoff between achievable diversity and multiplexing gains of any transmission over $N_{t} \times N_{r}$ MIMO channels.

The DMT formulated in [3] for uncorrelated Rayleigh MIMO channels represents an asymptotic framework as the signal to noise ratio (SNR) tends to infinity. Furthermore, spatial correlation does not affect the asymptotic DMT. It only degrades outage probability [4]. Space-time block codes (STBCs) for MIMO systems are commonly designed according to the asymptotic rank-determinant criteria [2]. A nonzero minimum determinant is sufficient to reach the frontier of the asymptotic DMT [5]. Recently, several papers have noted that STBCs designed at high (asymptotic) SNRs are not effective at low to medium SNRs [6-8] where practical communication systems operate. Moreover, we have shown in [7,8] that design parameters of STBCs are SNR-dependent and therefore that adaptive STBCs are more effective for practical communication systems.

Motivated by the characterization of the tradeoff between diversity and multiplexing gains at finite SNRs, the finiteSNR DMT has been proposed in $[9,10]$. This finite-SNR DMT could provide new insights on how to design MIMO systems particularly suited to the propagation environment and optimized for realistic SNRs. In [9, 10], Narasimhan has pointed out that the exact form of the outage probability and therefore the finite-SNR DMT for any $N_{t} \times N_{r}$ MIMO channels are not tractable. Consequently, estimates of the finite-SNR DMT are usually derived, e.g., in [9-13]. In some special cases, the exact expression of the outage probability and the finiteSNR DMT can be derived. To the best of our knowledge, the exact expression of the finite-SNR DMT is derived only for $2 \times 2$ MIMO systems with uncorrelated Rayleigh fading [14], and for multiple-input single-output (MISO) and single-input multiple-output (SIMO) systems with uncorrelated [15] and correlated [13] Rayleigh fading. Recently, we have derived in [16] the exact outage probability and the finite-SNR DMT for uncorrelated Rayleigh fading MIMO channels with dual antennas. However, in real scenarios, channel coefficients are not always independent, e.g., when the spacing between antennas is insufficient [17]. The spatial correlation may lead to a substantial degradation of the achievable diversity gains at finite SNRs $[10,12,13]$. In this paper, inspired from our work in [16], we derive the exact outage probability and finiteSNR DMT for Rayleigh fading channels with dual correlated antennas, i.e., $N_{t} \times 2$ and $2 \times N_{r}$ MIMO systems.

The rest of the paper is organized as follows. Section II introduces the system model and the definitions of diversity and multiplexing gains at finite SNR. The exact outage probability and finite-SNR DMT for spatially correlated Rayleigh fading MIMO channels with dual antennas are derived in Section III. Outage probability and finite-SNR DMT results are provided in Section IV. Section V concludes the paper. 


\section{SySTEM MODEL AND DEFINITIONS}

We consider a MIMO system with $N_{t}$ transmit antennas and $N_{r}$ receive antennas, operating over a flat Rayleigh fading channel. A perfect channel state information (CSI) is assumed at the receiver, but not at the transmitter. A quasi-static fading is assumed where the channel remains constant over one spacetime coding block of length $T$ and changes independently across blocks.

The channel input-output relation is given by:

$$
\mathbf{Y}=\mathbf{H X}+\mathbf{N}
$$

where $\mathbf{X}_{\left[N_{t} \times T\right]}$ is the transmitted space-time block code, $\mathbf{Y}_{\left[N_{r} \times T\right]}$ is the matrix of received $N_{t}$ noisy signals over $T$ channel uses and $\mathbf{N}_{\left[N_{r} \times T\right]}$ is the matrix of additive complex white Gaussian noise with independent and identically distributed (i.i.d.) entries and probability density function (pdf) $\sim C N\left(0, \sigma^{2}\right)$. The MIMO spatially correlated Rayleigh channel is modeled by the matrix $\mathbf{H}$ which can be factorized as:

$$
\mathbf{H}=\mathbf{R}_{\mathrm{Rx}}^{1 / 2} \mathbf{H}_{w} \mathbf{R}_{\mathrm{Tx}}^{1 / 2}
$$

where $\mathbf{H}_{w}$ is the $N_{r} \times N_{t}$ matrix modeling the uncorrelated Rayleigh fading channel with i.i.d. circularly symmetric complex Gaussian random variables entries and pdf $\sim C N(0,1)$, $\mathbf{R}_{\mathrm{Rx}}$ and $\mathbf{R}_{\mathrm{Tx}}$ are the $N_{r} \times N_{r}$ and $N_{t} \times N_{t}$ correlation matrices at the receiver and the transmitter respectively. The channel model in (2) is known in the literature as the Kronecker model and it is usually used to model the spatially correlated channel, e.g., in $[4,10-13,18-20]$.

In [3], conventional asymptotic definitions of multiplexing and diversity gains for a MIMO channel are given by:

$$
\begin{gathered}
r_{\text {asymptotic }}=\lim _{\rho \rightarrow \infty} \frac{R}{\log \rho} \\
d_{\text {asymptotic }}=-\lim _{\rho \rightarrow \infty} \frac{\log P_{\text {out }}}{\log \rho}
\end{gathered}
$$

where $r_{\text {asymptotic }}$ and $d_{\text {asymptotic }}$ represent the asymptotic multiplexing and diversity gains respectively, $\rho$ is the average SNR per receive antenna, $R$ is the MIMO system data rate and $P_{\text {out }}$ is the outage probability. As no CSI is available at the transmitter, an equal power across transmit antennas is adopted and $P_{\text {out }}$ is defined as:

$$
P_{\text {out }}=\operatorname{Pr}[I \leq R]
$$

where $I$ is the mutual information between received and transmitted signals over the MIMO channel.

The asymptotic diversity-multiplexing or data ratereliability tradeoff represents the upper bound achievable by any transmission over a $N_{t} \times N_{r}$ MIMO system. The asymptotic DMT has been established in [3] for uncorrelated Rayleigh fading channels. Later, [4] has proved that the spatial correlation does not affect the asymptotic DMT. The asymptotic DMT is given by the piece-wise linear function connecting the points $\left(k, d^{*}(k)\right)$ where $d^{*}(k)$ is given by:

$$
d^{*}(k)=\left(N_{t}-k\right)\left(N_{r}-k\right) ; k=1, \cdots, \min \left(N_{t}, N_{r}\right)
$$

At finite SNR $\rho$, the multiplexing gain $r$ is defined as the ratio of the system data rate $R$ to the capacity of an additive white Gaussian noise (AWGN) channel with array gain $G$ [10]:

$$
r=\frac{R}{\log (1+G \rho)}
$$

where the array gain is chosen such that $G=N_{r}$ in order to have a fair comparison of diversity and outage performance across different combinations of $N_{t}$ and $N_{r}$ antennas at low to medium SNRs [9, 10].

When considering a capacity-approaching channel code in the communication chain, the block-error probability is well approximated by the channel outage at finite SNR $[9,10]$. Therefore, the diversity gain $d(r, \rho)$ of a system with a fixed multiplexing gain $r$ at $\operatorname{SNR} \rho$ is defined by the negative slope of the log-log curve of the outage probability versus SNR, leading to:

$$
d(r, \rho)=-\frac{\partial \log P_{\text {out }}(r, \rho)}{\partial \log \rho}=-\frac{\rho}{P_{\text {out }}(r, \rho)} \frac{\partial P_{\text {out }}(r, \rho)}{\partial \rho}
$$

This definition is important for system design as the diversity gain at a particular operating SNR can be used to estimate the additional SNR required to reach a target outage probability, for a given data rate represented by the multiplexing gain.

In the sequel, we derive the exact finite-SNR DMT for systems with dual correlated transmit and/or receive antennas over Rayleigh fading channels.

\section{Computation OF FINITE-SNR DMT}

\section{A. Mutual information $p d f$}

In this section, we derive an analytical expression for the pdf of the mutual information between received and transmitted signals for $N_{t} \times 2$ and $2 \times N_{r}$ MIMO systems. By assuming that $\mathbf{X}$ is a zero-mean white complex Gaussian random variable, the MIMO mutual information $I$ conditioned on the channel realization $\mathbf{H}$ is given by [21]:

$$
\begin{aligned}
I & =\log \operatorname{det}\left(\mathbf{I}_{N_{r}}+\frac{\rho}{N_{t}} \mathbf{H} \mathbf{H}^{H}\right) \\
& =\log \operatorname{det}\left(\mathbf{I}_{N_{t}}+\frac{\rho}{N_{t}} \mathbf{H}^{H} \mathbf{H}\right)
\end{aligned}
$$

where the superscript ${ }^{H}$ stands for conjugate transpose.

As in [10-13], we focus on the spatial correlation only on one side, i.e., the transmitter side or the receiver side. A typical example of such a system is a communication between a mobile station with dual antennas and a base station with $n$ antennas. Here, the antennas at the base station can be spaced sufficiently far apart to achieve uncorrelation but, due to physical size constraints, it is more difficult to space antennas far apart at the mobile station. Indeed, when dual transmit antennas are correlated, the channel matrix $\mathbf{H}$ is equal to $\mathbf{H}_{w} \mathbf{R}_{\mathrm{Tx}}^{1 / 2}$, and when dual receive antennas are correlated $\mathbf{H}$ is equal to $\mathbf{R}_{\mathrm{Rx}}^{1 / 2} \mathbf{H}_{w}$. For this case, matrices $\mathbf{H} \mathbf{H}^{H}$ and $\mathbf{H}^{H} \mathbf{H}$ are central complex Wishart distributed and the pdf 


$$
\begin{gathered}
f^{\operatorname{corr}}(x, y)=\frac{\left(a_{1} a_{2}\right)^{n}\left(\frac{N_{t}}{\rho}\right)^{2 n-1}}{\left(a_{2}-a_{1}\right) \Gamma(n) \Gamma(n-1)} e^{x+y}\left(e^{x}-e^{y}\right)\left(e^{x}-1\right)^{n-2}\left(e^{y}-1\right)^{n-2} \\
\times\left[e^{-\frac{N_{t}}{\rho}\left(a_{1} e^{x}+a_{2} e^{y}-a_{1}-a_{2}\right)}-e^{-\frac{N_{t}}{\rho}\left(a_{1} e^{y}+a_{2} e^{x}-a_{1}-a_{2}\right)}\right] \\
f^{\operatorname{corr}}(I, y, \rho)=\frac{\left(a_{1} a_{2}\right)^{n}\left(\frac{N_{t}}{\rho}\right)^{2 n-1}}{\left(a_{2}-a_{1}\right) \Gamma(n) \Gamma(n-1)} e^{I}\left(e^{I-y}-e^{y}\right)\left(e^{I-y}-1\right)^{n-2}\left(e^{y}-1\right)^{n-2} \\
\times\left[e^{-\frac{N_{t}}{\rho}\left(a_{1} e^{I-y}+a_{2} e^{y}-a_{1}-a_{2}\right)}-e^{-\frac{N_{t}}{\rho}\left(a_{1} e^{y}+a_{2} e^{I-y}-a_{1}-a_{2}\right)}\right], \\
g^{\operatorname{corr}}(y, I, \rho)=\left(e^{I-y}-e^{y}\right)\left(e^{I-y}-1\right)^{n-2}\left(e^{y}-1\right)^{n-2} \\
\times\left[e^{-\frac{N_{t}}{\rho}\left(a_{1} e^{I-y}+a_{2} e^{y}-a_{1}-a_{2}\right)}-e^{-\frac{N_{t}}{\rho}\left(a_{1} e^{y}+a_{2} e^{I-y}-a_{1}-a_{2}\right)}\right] . \\
\frac{\partial g^{\operatorname{corr}}(y, I, \rho)}{\partial \rho}=\left(e^{I-y}-e^{y}\right)\left(e^{I-y}-1\right)^{n-2}\left(e^{y}-1\right)^{n-2} \\
\quad\left[\frac{N_{t}}{\rho^{2}}\left(a_{1} e^{I-y}+a_{2} e^{y}-a_{1}-a_{2}\right) e^{-\frac{N_{t}}{\rho}\left(a_{1} e^{I-y}+a_{2} e^{y}-a_{1}-a_{2}\right)}\right] \\
\left.-\frac{N_{t}}{\rho^{2}}\left(a_{2} e^{I-y}+a_{1} e^{y}-a_{1}-a_{2}\right) e^{-\frac{N_{t}}{\rho}\left(a_{2} e^{I-y}+a_{1} e^{y}-a_{1}-a_{2}\right)}\right]
\end{gathered}
$$

of their eigenvalues ${ }^{1}$ can be found in [22]. Let us define $m \triangleq \min \left(N_{t}, N_{r}\right)$ and $n \triangleq \max \left(N_{t}, N_{r}\right)$. For $m=2$, the joint pdf of the two nonzero ordered eigenvalues $\lambda_{1}$ and $\lambda_{2}$ $\left(\lambda_{1} \geq \lambda_{2}\right)$ is given by [22]:

$$
\begin{aligned}
& f^{\text {corr }}\left(\lambda_{1}, \lambda_{2}\right)=\frac{\left(a_{1} a_{2}\right)^{n}\left(\lambda_{1} \lambda_{2}\right)^{n-2}\left(\lambda_{1}-\lambda_{2}\right)}{\left(a_{2}-a_{1}\right) \Gamma(n) \Gamma(n-1)} \\
& \quad \times\left[e^{-\left(a_{1} \lambda_{1}+a_{2} \lambda_{2}\right)}-e^{-\left(a_{1} \lambda_{2}+a_{2} \lambda_{1}\right)}\right]
\end{aligned}
$$

where $a_{1}$ and $a_{2}$ are the ordered eigenvalues $\left(a_{2} \geq a_{1}\right)$ of $\mathbf{R}_{\mathrm{Rx}}^{-1}$ (downlink) or $\mathbf{R}_{\mathrm{Tx}}^{-1}$ (uplink) and $\Gamma(x)$ is the gamma function defined by $\int_{0}^{\infty} t^{x-1} e^{-t} \mathrm{~d} t$.

Therefore, the mutual information $I$ is expressed by:

$$
I=\log \left(1+\lambda_{1} \frac{\rho}{N_{t}}\right)+\log \left(1+\lambda_{2} \frac{\rho}{N_{t}}\right) .
$$

Let us define new variables $x$ and $y$ as:

$$
x \triangleq \log \left(1+\lambda_{1} \frac{\rho}{N_{t}}\right) \text { and } y \triangleq \log \left(1+\lambda_{2} \frac{\rho}{N_{t}}\right) .
$$

Lemma 1: The joint pdf of $x$ and $y$ denoted by $f^{\text {corr }}(x, y)$ is given in (13) at the top of the page, where $x \geq y \geq 0$.

Proof: The joint pdf of $x$ and $y$ can be derived from (10) by [23],

$$
f^{\text {corr }}(x, y)=f^{\text {corr }}\left(\lambda_{1}, \lambda_{2}\right) \frac{\partial \lambda_{1}}{\partial x} \frac{\partial \lambda_{2}}{\partial y}
$$

where

$$
\lambda_{1} \triangleq \frac{N_{t}}{\rho}\left(e^{x}-1\right) \text { and } \lambda_{2} \triangleq \frac{N_{t}}{\rho}\left(e^{y}-1\right) .
$$

By replacing $\lambda_{1}$ and $\lambda_{2}$ in (10) and computing

$$
\frac{\partial \lambda_{1}}{\partial x}=\frac{e^{x} N_{t}}{\rho} \text { and } \frac{\partial \lambda_{2}}{\partial y}=\frac{e^{y} N_{t}}{\rho} .
$$

we obtain (13).

\footnotetext{
${ }^{1}$ We noted that the case of spatial correlation at both transmitter and receiver sides leads to a more complex form of the pdf for the two nonzero ordered eigenvalues $\lambda_{1}$ and $\lambda_{2}$ [22]. Therefore, the exact finite-SNR DMT is not
} easily tractable for this case.
From the definition in (12) the mutual information between received and transmitted signals is $I=x+y$. The joint pdf of $I$ and $y, f^{\text {corr }}(I, y, \rho)$, is then given in (17) at the top of the page, where $I / 2 \geq y \geq 0$.

Lemma 2: At SNR $\rho$, and after integration of (17), the pdf of the mutual information between received and transmitted signals for a MIMO system with $n$ uncorrelated antennas and dual correlated antennas is given by:

$$
\begin{aligned}
& f^{\mathrm{corr}}(I, \rho)=\frac{\left(a_{1} a_{2}\right)^{n}\left(\frac{N_{t}}{\rho}\right)^{2 n-1}}{\left(a_{2}-a_{1}\right) \Gamma(n) \Gamma(n-1)} e^{I} \\
& \quad \times \int_{0}^{I / 2} g^{\operatorname{corr}}(y, I, \rho) \mathrm{d} y,
\end{aligned}
$$

where $g^{\text {corr }}(y, I, \rho)$ is expressed in (19) at the top of the page.

For each value of $I$ and $\rho$, the expression $\int_{0}^{I / 2} g(y, I, \rho) \mathrm{d} y$ can be computed by numerical integration.

\section{B. Outage probability}

Theorem 1: For each SNR $\rho$ and multiplexing gain $r$, the exact outage probability $P_{\text {out }}^{\text {corr }}(r, \rho)$ of a Rayleigh MIMO channel with $n$ uncorrelated antennas and dual correlated antennas is given from (18) by:

$$
\begin{aligned}
P_{\text {out }}^{\text {corr }}(r, \rho) & =\operatorname{Pr}[I \leq R] \\
& =\int_{0}^{R} f^{\text {corr }}(I, \rho) \mathrm{d} I \\
& =\frac{\left(a_{1} a_{2}\right)^{n}\left(\frac{N_{t}}{\rho}\right)^{2 n-1}}{\left(a_{2}-a_{1}\right) \Gamma(n) \Gamma(n-1)} \\
& \times \int_{0}^{r \log (1+G \rho)} e^{I}\left(\int_{0}^{I / 2} g^{\text {corr }}(y, I, \rho) \mathrm{d} y\right) \mathrm{d} I
\end{aligned}
$$

(20) is computed by numerical integration. This outage probability describes the performance of MIMO systems with dual correlated antennas and its slope defines the diversity gain at a given multiplexing gain $r$.

\section{Analytical finite-SNR DMT}

Theorem 2: The derivative of the outage probability of a Rayleigh MIMO channel with $n$ uncorrelated antennas and dual correlated antennas $\frac{\partial P_{\text {out }}^{\text {corr }}(r, \rho)}{\partial \rho}$ is given by:

$$
\frac{\partial P_{\text {out }}^{\text {corr }}(r, \rho)}{\partial \rho}=A_{1}^{\text {corr }}(r, \rho)+A_{2}^{\text {corr }}(r, \rho)+A_{3}^{\text {corr }}(r, \rho)
$$




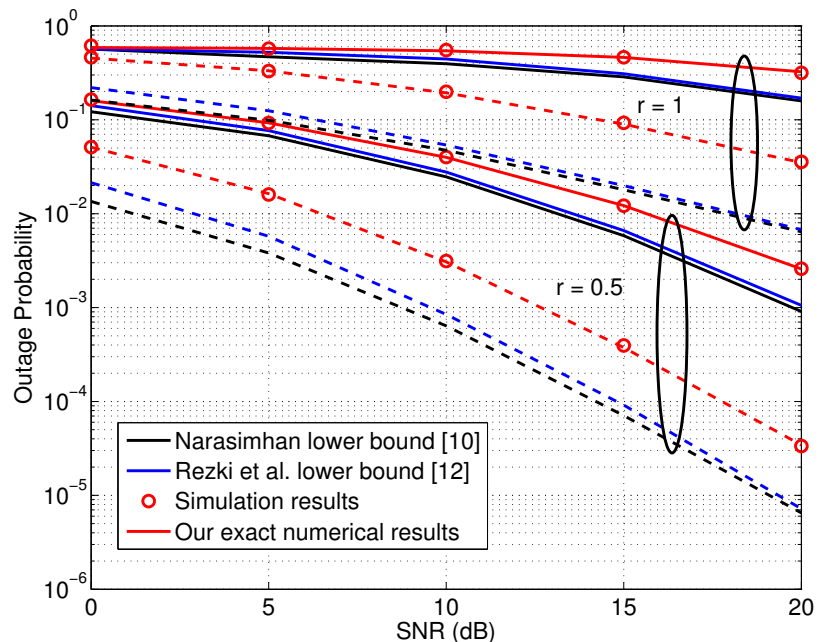

Fig. 1. Outage probability for different multiplexing gains $r=0.5,1$; $N_{t}=N_{r}=2$, uncorrelated and correlated $\left(\rho_{t}=0.0043+j 0.9789\right)$ flat Rayleigh fading with dashed and solid lines respectively.

where

$$
\begin{gathered}
A_{1}^{\text {corr }}(r, \rho)=\frac{(-2 n+1)\left(N_{t}\right)^{2 n-1} \frac{1}{\rho^{2 n}}\left(a_{1} a_{2}\right)^{n}}{\left(a_{2}-a_{1}\right) \Gamma(n) \Gamma(n-1)} \\
\quad \times \int_{0}^{r \log (1+G \rho)} e^{I}\left(\int_{0}^{I / 2} g^{\text {corr }}(y, I, \rho) \mathrm{d} y\right) \mathrm{d} I \\
=-\frac{2 n-1}{\rho} P_{\text {out }}(r, \rho), \\
A_{2}^{\text {corr }}(r, \rho)=\frac{\left(a_{1} a_{2}\right)^{n}\left(\frac{N_{t}}{\rho}\right)^{2 n-1}}{\left(a_{2}-a_{1}\right) \Gamma(n) \Gamma(n-1)} \frac{r G}{1+G \rho} e^{r \log (1+G \rho)} \\
\quad \times\left(\int_{0}^{r \log (1+G \rho) / 2} g^{\text {corr }}(y, r \log (1+G \rho), \rho) \mathrm{d} y\right),
\end{gathered}
$$

and

$$
\begin{aligned}
& A_{3}^{\text {corr }}(r, \rho)=\frac{\left(a_{1} a_{2}\right)^{n}\left(\frac{N_{t}}{\rho}\right)^{2 n-1}}{\left(a_{2}-a_{1}\right) \Gamma(n) \Gamma(n-1)} \\
& \quad \times \int_{0}^{r \log (1+G \rho)} e^{I}\left(\int_{0}^{I / 2} \frac{\partial g^{\text {corr }}(y, I, \rho)}{\partial \rho} \mathrm{d} y\right) \mathrm{d} I
\end{aligned}
$$

where $\frac{\partial g^{\text {corr }}(y, I, \rho)}{\partial \rho}$ is given in (25) at the top of the previous page.

Proof: The proof is presented in Appendix A.

The above expressions can also be computed using numerical integration. Using the outage probability given in (20) and its derivative given in (21), the finite-SNR DMT for a Rayleigh MIMO channel with $n$ uncorrelated antennas and dual correlated antennas is now easily computed using (8).

\section{NUMERICAL RESULTS}

In this section, numerical results are given. We consider as in [10-13] that the dual transmit antennas are correlated. The transmit correlation matrix is then given by:

$$
\mathbf{R}_{\mathrm{Tx}}=\left[\begin{array}{cc}
1 & \rho_{t} \\
\rho_{t}^{*} & 1
\end{array}\right]
$$

In order to assess the accuracy of our derived DMT, we consider, as in [10], $\rho_{t}$ equal to $0.0043+j 0.9789$ for the high spatial correlation case. Note that the results obtained in [16] for uncorrelated channels can be easily computed from the derived equations by letting $\rho_{t} \rightarrow 0$.

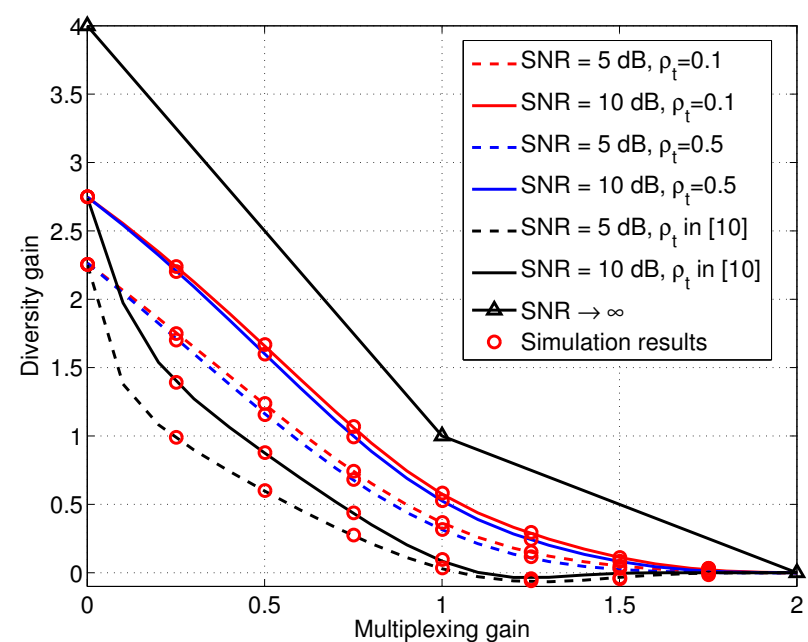

Fig. 2. Asymptotic and exact finite-SNR DMT curves for $2 \times 2$ correlated flat Rayleigh MIMO channel with low $\rho_{t}=0.1$ (red curves), medium $\rho_{t}=0.5$ (blue curves) and high $\rho_{t}=0.0043+j 0.9789$ (black curves) correlations at $\mathrm{SNR}=5 \mathrm{~dB}$ with dashed lines and SNR $=10 \mathrm{~dB}$ with solid lines.

Fig. 1 depicts the outage probability for multiplexing gain $r$ equal to 0.5 and 1 as a function of SNR, for both uncorrelated and correlated flat Rayleigh fading channels with dashed and solid lines respectively. Our outage probability curves computed by (20) are identical to the ones obtained by Monte Carlo simulations, which shows that our exact results are very accurate. It can also be observed that the lower bounds on the outage probability derived by Narasimhan [10] and by Rezki et. al [12] are far from exact values for both uncorrelated and correlated channels. Furthermore, Fig. 1 shows that a lower outage probability is reached in the uncorrelated case. At $r=0.5$ and $r=1$, a steeper slope of the outage probability curves is observed for the uncorrelated channel with respect to the correlated channel at low to moderate SNRs. Thus, at the same multiplexing gain $r$ and at finite SNR, one can expect that a higher diversity gain is obtained for the uncorrelated channel compared to the correlated channel.

Fig. 2 depicts the exact finite-SNR DMT curves for $N_{r}=$ $n=2$ at SNR $=5$ and $10 \mathrm{~dB}$ over correlated flat Rayleigh channel with low $\rho_{t}=0.1$, medium $\rho_{t}=0.5$ and high $\rho_{t}=0.0043+j 0.9789$ correlations. For all cases, our numerical results are identical to the simulation results obtained by Monte Carlo simulations. In addition, the upper bounds on the finite-SNR DMT derived by Narasimhan [10] and by Rezki et. al [12], not shown for figure clarity, overestimate the achievable finite-SNR DMT. At $\rho_{t}=0.1$, almost the same finite-SNR DMT is obtained for both uncorrelated [16] and correlated channels. As expected based on outage probability curves, the diversity gain decreases for correlated channels with respect to uncorrelated channels. Moreover, when the correlation coefficient increases, the diversity gain decreases. However, the diversity gain is only slightly degraded at low to moderate values of spatial correlation. On the contrary, the diversity gain is severely degraded at high correlations. 


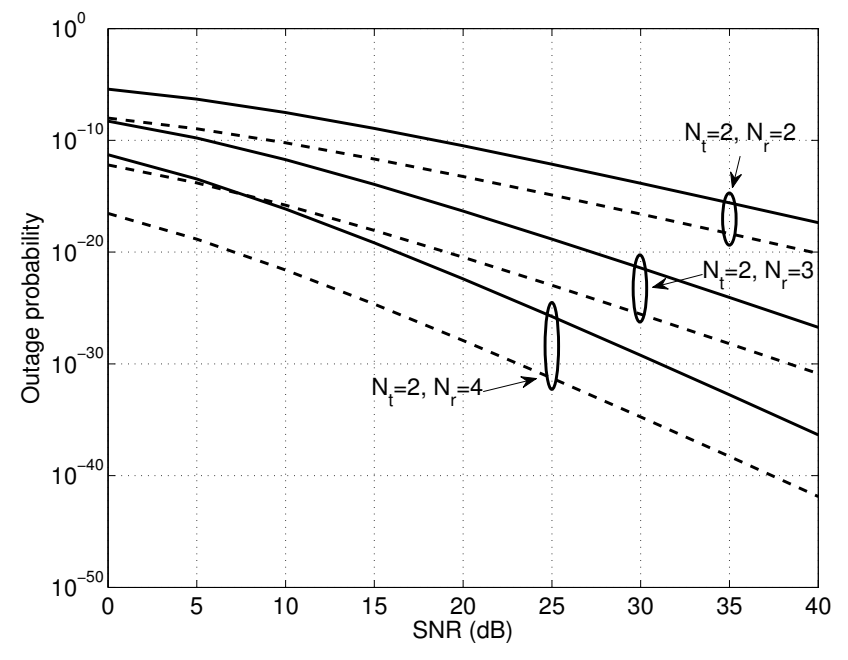

Fig. 3. Outage probability for MIMO systems with multiplexing gain $r \rightarrow 0$; $2 \times 2,2 \times 3$ and $2 \times 4$, in uncorrelated and correlated $\left(\rho_{t}=0.0043+j 0.9789\right)$ flat Rayleigh fading channels (dashed and solid lines respectively).

Also, Fig. 2 shows that the maximum achievable diversity, i.e., the diversity gain for $r \rightarrow 0$, at finite SNR is the same regardless of the spatial correlation value and is equal to the one of uncorrelated channels. Similar conclusions have also been found in [12] based on their derived estimates of finiteSNR DMT.

Now let us consider a higher number of antennas $n \geq$ 3. Fig. 3 depicts the outage probability curves when the multiplexing gain $r$ tends to 0 . A high spatial correlation $\left(\rho_{t}=0.0043+j 0.9789\right)$ at the transmitter and several uncorrelated antennas $\left(N_{r}=2,3,4\right)$ at the receiver are considered. Outage probability curves define the achievable block-error probability by any transmission over $2 \times n$ uncorrelated and correlated MIMO channels. Furthermore, low outage probabilities can be easily assessed using our derived analytical equations while Monte Carlo simulation results are limited to outage probabilities higher than $10^{-6}$ because of simulation complexity [9-13]. Fig. 3 also proves that the exact maximum diversity at finite-SNR is the same for both uncorrelated and correlated channels. Indeed, at any SNR, the figure shows that the outage probability curves for uncorrelated and correlated channels have the same slope and therefore the same maximum diversity, with a SNR loss for the correlated channels with respect to the uncorrelated channels. Indeed, lower outage probabilities can be achieved for the uncorrelated case. The SNR loss between uncorrelated and correlated channels is shown to increase with the increase of the number of receive antennas.

Finally, we investigate the effect of increasing the number of antennas on the finite-SNR DMT. Fig. 4 depicts, with dashed lines for a low correlation $\rho_{t}=0.1$ and solid lines for a high correlation $\rho_{t}=0.0043+j 0.9789$, the exact finite-SNR DMT curves for $2 \times 2$ and $2 \times 5$ correlated flat Rayleigh MIMO channels at various SNRs. This figure shows that the diversity loss between finite-SNR DMT for

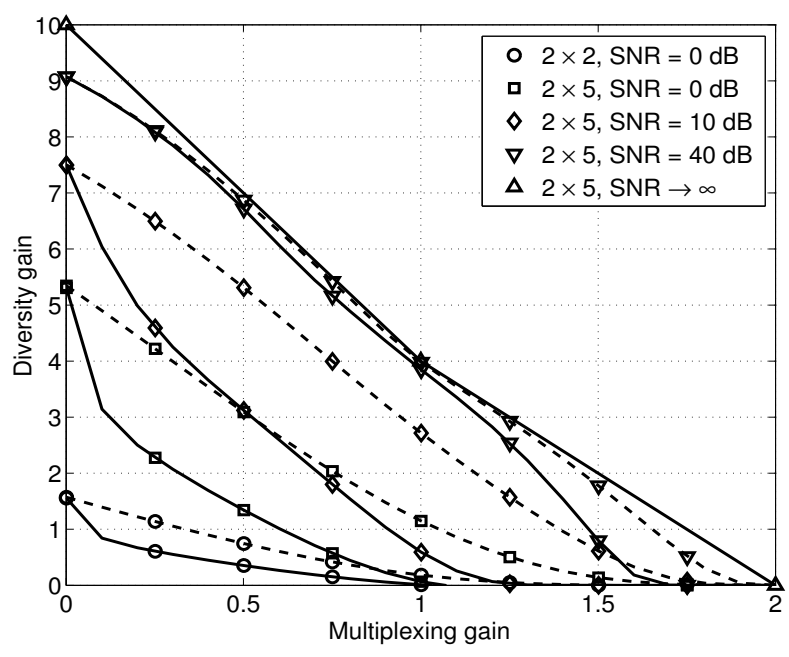

Fig. 4. Exact finite-SNR DMT curves for $2 \times 2$ and $2 \times 5$ correlated flat Rayleigh MIMO channel with a low $\rho_{t}=0.1$ and high $\rho_{t}=0.0043+$ $j 0.9789$ correlation at various SNRs (dashed and solid lines respectively).

low and high spatial correlation curves increases with the increase of the number of receive antennas. Moreover, this diversity loss decreases with the SNR increase especially for low multiplexing gains. When $r \rightarrow 0$, the same maximum diversity is achieved regardless of the spatial correlation value as proven above based on Fig. 3 .

Also, all presented finite-SNR DMT curves show that achievable diversity gains at finite SNR are significantly lower than asymptotic values. A convergence of the finite-SNR DMT to the asymptotic DMT is obtained at high SNRs.

\section{CONCLUSIONS}

In this paper, we have derived the exact outage probability and finite-SNR diversity-multiplexing tradeoff for Rayleigh MIMO channels with dual correlated antennas. Actually, achievable diversity gains at realistic SNRs are significantly lower than asymptotic values for SNR $\rightarrow \infty$. The finiteSNR DMT characterizes the system at operational SNRs where available diversity gains are computed. While finiteSNR DMT and outage probabilities are usually only estimated, we have shown that the numerical results of our derived outage probability and finite-SNR DMT are identical to those obtained using Monte Carlo simulations. Also, it has been shown that the outage probability and the finite-SNR DMT degrade as the spatial correlation increases. They are only slightly degraded at low to moderate spatial correlations while important penalties are observed at high spatial correlations. However, we have proven that the spatial correlation does not affect the maximum diversity gain at finite SNRs. Afterwards, the SNR loss between outage probability curves, and the diversity loss between finite-SNR DMT curves, for low and high spatial correlations are shown to increase with the increase of the number of antennas. Conventionally, STCs for MIMO systems are designed to achieve the asymptotic DMT frontier and therefore are not efficient at realistic SNRs. This finiteSNR DMT could provide new insights on how to design STCs 
for practical MIMO systems optimized at realistic SNRs and propagation environments.

\section{APPENDIX A}

\section{PROOF OF THEOREM 2}

The Leibniz integral rule is used to compute the derivative of an integral and is expressed by [23]:

$$
\begin{aligned}
& \frac{\partial}{\partial \rho} \int_{a(\rho)}^{b(\rho)} f(I, \rho) \mathrm{d} I=\int_{a(\rho)}^{b(\rho)} \frac{\partial f(I, \rho)}{\rho} \mathrm{d} I \\
& \quad+f(b(\rho), \rho) \frac{\partial b(\rho)}{\partial \rho}-f(a(\rho), \rho) \frac{\partial a(\rho)}{\partial \rho}
\end{aligned}
$$

In our case,

$$
P_{\text {out }}^{\text {corr }}(r, \rho)=\int_{0}^{R} f^{\text {corr }}(I, \rho) \mathrm{d} I
$$

where

$$
\begin{aligned}
& f^{\mathrm{corr}}(I, \rho)=\frac{\left(a_{1} a_{2}\right)^{n}\left(\frac{N_{t}}{\rho}\right)^{2 n-1}}{\left(a_{2}-a_{1}\right) \Gamma(n) \Gamma(n-1)} e^{I} \\
& \quad \times \int_{0}^{I / 2} g^{\mathrm{corr}}(y, I, \rho) \mathrm{d} y
\end{aligned}
$$

Afterwards,

$$
\frac{\partial P_{\text {out }}^{\text {corr }}(r, \rho)}{\partial \rho}=\frac{\partial}{\partial \rho} \int_{0}^{R} f^{\text {corr }}(I, \rho) \mathrm{d} I
$$

can be computed using the Leibniz integral rule where $a(\rho)=$ 0 and $b(\rho)=R=r \log (1+G \rho)$. Therefore,

$$
f^{\mathrm{corr}}(a(\rho), \rho) \frac{\partial a(\rho)}{\partial \rho}=0,
$$

and

$$
\begin{aligned}
\frac{\partial P_{\text {out }}^{\text {cort }}(r, \rho)}{\partial \rho} & =\int_{a(\rho)}^{b(\rho)} \frac{\partial f^{\text {corr }}(I, \rho)}{\rho} \mathrm{d} I+f^{\text {corr }}(b(\rho), \rho) \frac{\partial b(\rho)}{\partial \rho} \\
& =A_{1}^{\text {corr }}(r, \rho)+A_{2}^{\text {corr }}(r, \rho)+A_{3}^{\text {corr }}(r, \rho) .
\end{aligned}
$$

Indeed,

$$
\begin{gathered}
f^{\text {corr }}(b(\rho), \rho) \frac{\partial b(\rho)}{\partial \rho}=f^{\text {corr }}(r \log (1+G \rho), \rho) \frac{r G}{1+G \rho} \\
=\frac{\left(a_{1} a_{2}\right)^{n}\left(\frac{N_{t}}{\rho}\right)^{2 n-1}}{\left(a_{2}-a_{1}\right) \Gamma(n) \Gamma(n-1)} \frac{r G}{1+G \rho} e^{r \log (1+G \rho)} \\
\times\left(\int_{0}^{r \log (1+G \rho) / 2} g^{\operatorname{corr}}(y, r \log (1+G \rho), \rho) \mathrm{d} y\right) \\
=A_{2}^{\text {corr }}(r, \rho),
\end{gathered}
$$

and

$$
\begin{gathered}
\int_{a(\rho)}^{b(\rho)} \frac{\partial f^{\text {corr }}(I, \rho)}{\rho} \mathrm{d} I= \\
{\left[\begin{array}{c}
\frac{\left(a_{1} a_{2}\right)^{n}\left(\frac{N_{t}}{\rho}\right)^{2 n-1}}{\left(a_{2}-a_{1}\right) \Gamma(n) \Gamma(n-1)} \\
\left.\times \int_{0}^{r \log (1+G \rho)} e^{I}\left(\int_{0}^{I / 2} \frac{\partial g^{\operatorname{corr}}(y, I, \rho)}{\partial \rho} \mathrm{d} y\right) \mathrm{d} I\right] \\
+ \\
{\left[\begin{array}{c}
\frac{(-2 n+1)\left(N_{t}\right)^{2 n-1} \frac{1}{\rho^{2 n}}\left(a_{1} a_{2}\right)^{n}}{\left(a_{2}-a_{1}\right) \Gamma(n) \Gamma(n-1)} \\
\times \int_{0}^{r \log (1+G \rho)} e^{I}\left(\int_{0}^{I / 2} g^{\text {corr }}(y, I, \rho) \mathrm{d} y\right) \mathrm{d} I
\end{array}\right]} \\
=A_{3}^{\text {corr }}(r, \rho)+A_{1}^{\text {corr }}(r, \rho) .
\end{array}\right.}
\end{gathered}
$$

\section{REFERENCES}

[1] P. Wolniansky, G. Foschini, G. Golden, and R. Valenzuela, "V-BLAST: an architecture for realizing very high data rates over the rich-scattering wireless channel," in Proc. IEEE Int. Symp. on Signals, Syst. and Electronics (ISSSE), Sep. 1998.

[2] V. Tarokh, N. Seshadri, and A. Calderbank, "Space-time codes for high data rate wireless communications: Performance criterion and code construction," IEEE Trans. Inf. Theory, vol. 44, no. 2, pp. 744-765, Mar. 1998.

[3] L. Zheng and D. Tse, "Diversity and multiplexing: a fundamental tradeoff in multiple-antenna channels," IEEE Trans. Inf. Theory, vol. 49, no. 5, pp. 1073-1096, May 2003.

[4] W. Chang, S.-Y. Chung, and Y. Lee, "Diversity-multiplexing tradeoff in rank-deficient and spatially correlated MIMO channels," in Proc. IEEE Int. Symp. Inf. Theory (ISIT), Jul. 2006, pp. 1144-1148.

[5] P. Elia, K. Kumar, S. Pawar, P. Kumar, and H.-F. Lu, "Explicit spacetime codes achieving the diversity-multiplexing gain tradeoff," IEEE Trans. Inf. Theory, vol. 52, no. 9, pp. 3869-3884, Sep. 2006.

[6] A. Lozano and N. Jindal, "Transmit diversity vs. spatial multiplexing in modern MIMO systems," IEEE Trans. Wireless Commun., vol. 9, no. 1, pp. 186 - 197, Jan. 2010.

[7] A. El Falou, C. Langlais, C. Abdel Nour, and C. Douillard, "Adaptive trace-orthonormal STBC for MIMO system with capacity approaching FEC codes," in Proc. IEEE Vehicular Technology Conference (VTC), Sep. 2012.

[8] - "Low ML-detection complexity, adaptive $2 \times 2$ STBC, with powerful FEC codes," in Proc. IEEE Symp. on Turbo Codes \& Iterative Information Processing, Aug. 2012.

[9] R. Narasimhan, "Finite-SNR diversity performance of rate-adaptive MIMO systems," in Proc. IEEE Global Telecommun. Conf. (GLOBECOM), Dec. 2005.

[10] —, "Finite-SNR diversity-multiplexing tradeoff for correlated Rayleigh and Rician MIMO channels," IEEE Trans. Inf. Theory, vol. 52, no. 9, pp. $3965-3979$, Sep. 2006.

[11] Z. Rezki, B. Cotruta, D. Haccoun, and F. Gagnon, "Finite diversity multiplexing tradeoff over spatially correlated channels," in Proc. IEEE Vehicular Technology Conference (VTC), Sep. 2006.

[12] Z. Rezki, D. Haccoun, F. Gagnon, and W. Ajib, "Impact of spatial correlation on the finite-SNR diversity-multiplexing tradeoff," IEEE Trans. Wireless Commun., vol. 7, no. 4, pp. 1184-1189, Apr. 2008.

[13] _ "Diversity-multiplexing tradeoff over correlated Rayleigh fading channels: a non-asymptotic analysis," Wirel. Commun. Mob. Comput., vol. 10, no. 2, pp. 188-199, Feb. 2010.

[14] H. Ebrahimzad and A. Mohammadi, "Diversity-multiplexing tradeoff in MIMO system with finite SNR," in European Conference on Wireless Technologies, Oct. 2007, pp. $146-149$.

[15] — "Diversity-multiplexing tradeoff in MISO/SIMO systems at finite SNR," in Proc. IEEE Vehicular Technology Conference (VTC), Apr. 2007, pp. $594-598$.

[16] A. El Falou, W. Hamouda, C. Langlais, C. Abdel Nour, and C. Douillard, "Finite-SNR diversity-multiplexing tradeoff for rayleigh MIMO channels," IEEE Commun. Lett., vol. 17, no. 4, pp. 753-756, Apr. 2013.

[17] J. Winters, J. Salz, and R. Gitlin, "The impact of antenna diversity on the capacity of wireless communication systems," IEEE Trans. Commun., vol. 42, no. 2, pp. 1740-1751, Apr. 1994.

[18] H. A. Saleh and W. Hamouda, "Performance of zero-forcing detectors over MIMO flat-correlated Ricean fading channels," IET Communications, vol. 3, no. 1, pp. 10-16, 2009.

[19] W. Hamouda and M. Aljerjawi, "A transmit diversity scheme using space-time spreading for DS-CDMA systems in rayleigh fading channels," in Proc. IEEE Vehicular Technology Conference (VTC), vol. 1, Sep. 2005, pp. 147-151.

[20] W. N. An and W. Hamouda, "Reduced complexity MIMO concatenated code in fading channels," Communications Letters, IEEE, vol. 15, no. 7, pp. 746-748, Jun. 2011.

[21] I. Telatar, "Capacity of multi-antenna Gaussian channels," Europ. Trans. Telecommun., vol. 10, pp. 585 - 595, 1999.

[22] T. Ratnarajah and R. Vaillancourt, "Quadratic forms on complex random matrices and multiple-antenna systems," IEEE Trans. Inf. Theory, vol. 51, no. 8, pp. 2976 - 2984, Aug. 2005.

[23] A. Papoulis and S. Pillai, Probability, random variable, and stochastic processes, Fourth edition. McGraw-Hill. Press, 2002. 